\title{
Dímero D e infarto do miocárdio em pacientes com COVID-19: uma revisão
}

\section{sistemática e meta-análise}

\author{
D-dimer and myocardial infarction in patients with COVID-19: a systematic review and meta- \\ analysis
}

Dímero D e infarto del miocardio en pacientes con COVID-19: revisión sistemática y metanálisis

Recebido: 04/01/2022 | Revisado: 11/01/2022 |Aceito: 18/01/2022 | Publicado: 19/01/2022

\author{
Andressa Nogueira Cardoso \\ ORCID: https://orcid.org/0000-0002-7332-7560 \\ Universidade Estadual do Ceará, Brasil \\ E-mail: andressa.nogueira@aluno.uece.br \\ Denis Francisco Gonçalves de Oliveira \\ ORCID: https://orcid.org/0000-0003-0412-7300 \\ Universidade Federal do Ceará, Brasil \\ E-mail: denisfgo@ufc.br \\ Sued Magalhães Moita \\ ORCID: https://orcid.org/0000-0001-7189-6199 \\ Universidade Estadual do Ceará, Brasil \\ E-mail: sued.moita@aluno.uece.br \\ Ivna Leite Reis \\ ORCID: https://orcid.org/0000-0003-3477-0823 \\ Universidade Estadual do Ceará, Brasil \\ E-mail: ivna.reis@aluno.uece.br \\ Karinne da Silva Assunção \\ ORCID: https://orcid.org/0000-0002-8346-1384 \\ Universidade Estadual do Ceará, Brasil \\ E-mail: karinne.assuncao@aluno.uece.br \\ Ana Paula Peres Martins Gomes \\ ORCID: https://orcid.org/0000-0003-2422-5127 \\ Centro Universitário Christus, Brasil \\ E-mail: anapaulac7s@hotmail.com \\ Sthefane Gomes Feitosa \\ ORCID: https://orcid.org/0000-0002-4254-7210 \\ Universidade Estadual do Ceará, Brasil \\ E-mail: sthefane.feitosa@uece.br
}

\begin{abstract}
Resumo
O presente estudo buscou identificar a evidência atual sobre a associação entre níveis de dímero D e a ocorrência de infarto do miocárdio (IM) em pacientes com COVID-19. Para isso, foi realizada uma revisão sistemática, utilizando as seguintes bases de dados: PubMed, Embase, Web of Science, Scopus, Livivo, e Google Scholar. Os critérios de inclusão adotados foram: estudos que investigaram a associação entre COVID-19 e IM; e que apresentaram dados de dímero D. Foram excluídos: estudos de revisão, com modelos animais, in vitro, e relatos de casos. Para a síntese dos resultados, utilizaram-se meta-análises para diferença média padronizada (DMP) referente aos níveis de dímero D. Dessa forma, foram incluídos 6 estudos nesta revisão sistemática apresentando duas abordagens metodológicas: 2 estudos investigaram casos de COVID-19 buscando identificar o desenvolvimento de infarto do miocárdio; e os outros 4 estudos avaliaram pacientes com infarto do miocárdio com o propósito de determinar aqueles pacientes que haviam apresentado COVID-19. Os resultados da DMP nas meta-análises para cada abordagem foram respectivamente: 0,86 [IC95\% (0,63 a 1,08), p < 0,0001, favorecendo o grupo que desenvolveu IM] e 2,08 [IC95\% (0,01 a 4,17), $\mathrm{p}=0,0512$, favorecendo quem havia apresentado COVID-19]. Por conseguinte, nesta revisão sistemática demonstramos que existe associação entre os níveis de dímero D em pacientes com COVID-19 e infarto do miocárdio. No entanto, esses resultados apresentaram evidência muito baixa na classificação GRADE, o que aponta a necessidade de mais estudos acerca da temática.
\end{abstract}

Palavras-chave: COVID-19; Dímero D; Infarto do miocárdio; SARS-CoV-2.

\section{Abstract}

This study aimed to identify current evidence on the association between D-dimer levels and the occurrence of myocardial infarction (MI) in patients with COVID-19. For this, a systematic review was performed, using the following databases: PubMed, Embase, Web of Science, Scopus, Livivo, and Google Scholar. We applied the 
following inclusion criteria: studies that investigated the association between COVID-19 and MI; and studies with Ddimer data. We exclude review studies, with animal models, in vitro, and case reports. For the synthesis of the results, we used a standardized mean difference (SDM) meta-analysis to pool the D-dimer data. Thus, 06 studies were included in this systematic review presenting two methodological approaches: 02 studies investigated cases of COVID-19, seeking to identify the development of myocardial infarction; and the other 04 studies evaluated patients with myocardial infarction to determine those patients who had presented COVID-19. The SDM results of the metaanalyses for each approach were respectively: 0.86 [95\% CI $(0.63$ to 1.08$), \mathrm{p}<0.0001$, favoring the group that developed MI] and 2.08 [95\% CI (-0.01 to 4.17), $\mathrm{p}=0.0512$, favoring those who had presented COVID-19]. Therefore, in this systematic review, we demonstrate an association between D-dimer levels in patients with COVID-19 and myocardial infarction. However, these results showed very low evidence in the GRADE classification, which indicates the need for more studies on the topic.

Keywords: COVID-19; D-dimer; Myocardial infarction; SARS-CoV-2.

\section{Resumen}

El presente estudio buscó identificar la evidencia actual sobre la asociación entre los niveles de dímero D y infarto del miocardio (IM) en pacientes con COVID-19. Para ello, se realizó una revisión sistemática, utilizando las bases de datos: PubMed, Embase, Web of Science, Scopus, Livivo y Google Scholar. Los criterios de inclusión adoptados fueron: estudios que investigaron la asociación entre COVID-19 e IM; y que presentaran datos del dímero D. Se excluyeron: estudios de revisión, con modelos animales, in vitro y reportes de casos. Para la síntesis de los resultados, se utilizaron metanálisis de diferencia de medias estandarizada (DME) con respecto a los niveles de dímero D. Así, se incluyeron 6 estudios en esta revisión sistemática que presentan dos enfoques metodológicos: 2 estudios investigaron casos de COVID-19, buscando identificar el desarrollo de infarto de miocardio; y los otros 4 estudios evaluaron pacientes con infarto de miocardio para determinar aquellos pacientes que habían presentado COVID-19. Los resultados de DME en los metanálisis para cada enfoque fueron respectivamente: 0,86 [IC95\% $(0,63$ a 1,08), p < 0,0001, a favor del grupo que desarrolló IM] y 2,08 [IC95\% (-0,01 a 4,17), p = 0.0512, favoreciendo a quienes habían presentado COVID-19]. Por tanto, en esta revisión sistemática demostramos que existe una asociación entre los niveles de dímero D en pacientes con COVID-19 e infarto de miocardio. Sin embargo, estos resultados arrojaron evidencia muy baja en la clasificación GRADE, lo que indica la necesidad de más estudios sobre el tema.

Palabras clave: COVID-19; Dímero D; Infarto del miocardio; SARS-CoV-2.

\section{Introdução}

A infecção respiratória aguda causada pelo SARS-CoV-2 se originou pela primeira vez em 2019 em Wuhan, China. Em seguida, a Organização Mundial da Saúde (OMS) declarou oficialmente a epidemia da doença coronavírus 2019 (COVID19) como uma emergência de saúde pública de interesse internacional (Shah et al., 2020).

As principais manifestações clínicas da doença são febre e sintomas do trato respiratório, porém a doença pode envolver outros sistemas e apresentar manifestações extra respiratórias, incluindo: cardíacas, gastrointestinais, hepáticas, renais, neurológicas, olfatórias, gustativas, oculares, cutâneas e hematológicas. Sobre as complicações cardíacas, os mecanismos envolvidos parecem ser decorrentes de lesão viral direta, hipoxemia, estado hemodinâmico instável com hipoperfusão, inflamação sistêmica aumentada, regulação negativa da enzima conversora de angiotensina 2 (ACE2), produção aumentada de catecolaminas endógenas e até mesmo toxicidade de medicamentos (Lai et al., 2020).

Nessa perspectiva, é importante buscar entender esses mecanismos envolvidos no desenvolvimento de eventos cardíacos em pacientes com COVID-19, e identificar marcadores capazes de predizer tais eventos. Dessa forma, emerge o papel clínico do dímero D. Sabe-se que este marcador tem se mostrado um bom preditor de progressão da COVID-19 e também de mortalidade nesses pacientes (Shah et al., 2020; Varikasuvu et al., 2021). Destaca-se ainda que estudo anterior à pandemia da COVID-19 mostrou que o nível elevado de dímero D está associado a eventos cardiovasculares subsequentes em homens aparentemente saudáveis e pacientes com angina instável (Choi et al., 2016).

Nesse sentido, a presente revisão sistemática buscou identificar a evidência atual sobre a associação entre níveis de dímero D e a ocorrência de infarto do miocárdio em pacientes com COVID-19. 


\section{Metodologia}

Para o relato da presente pesquisa utilizamos o Preferred Reporting Items for Systematic Reviews and Meta-Analyses (PRISMA) (Page et al., 2021). Vale destacar que o protocolo da revisão foi cadastrado no International Prospective Register of Systematic Reviews (PROSPERO) sob o número CRD42021272129.

\subsection{Critérios de Elegibilidade}

A pergunta que norteou a construção desta revisão foi a seguinte: existe associação entre os níveis de dímero D e a ocorrência de infarto do miocárdio em pacientes com COVID-19? Por conseguinte, adotou-se os seguintes critérios de inclusão: estudos que analisaram casos de COVID-19 e relataram os níveis de dímero D em pacientes que desenvolveram e naqueles que não desenvolveram infarto do miocárdio; e estudos que avaliaram pacientes com infarto do miocárdio e que descreveram os níveis de dímero D em pacientes que haviam apresentado COVID-19 e naqueles que não tiveram a doença viral. Ressalta-se que não restringimos o período de publicação, nem idioma. Em relação aos critérios de exclusão, foram removidos: estudos de revisão; estudos realizados em modelos animais ou in vitro; e relatos de casos.

\subsection{Fontes de Informação}

Executou-se as buscas nas seguintes bases de dados: PubMed, Embase, Web of Science, Scopus, Livivo e no Google Scholar (para a identificação de literatura cinzenta). Para o gerenciamento das referências e remoção de estudos duplicados utilizamos o software EndNote Web. É importante destacar que as buscas foram realizadas em 05 de agosto de 2021, e atualizadas em 29 de dezembro de 2021.

\subsection{Estratégia de Busca}

As estratégias de busca foram construídas com base nos seguintes descritores: "COVID-19", "fibrin fragment D", e "Myocardial Infarction". Utilizamos também termos semelhantes e os operadores booleanos "AND" e "OR". Para mais detalhes sobre a estratégia utilizada em cada base de dados consulte a Tabela 1.

\subsection{Seleção dos Estudos}

A seleção dos estudos foi realizada em duas fases. Na primeira, a seleção ocorreu por meio da leitura de título e resumo. Na segunda, foi necessário a leitura completa do artigo. Três autores realizaram a seleção em cada fase (ANC, KSA, SMM). Vale destacar que um quarto revisor (SGF) contribuiu para solucionar os casos de discordâncias entre os autores. Utilizou o software Rayyan (Ouzzani et al., 2016) para o gerenciamento das duas fases do processo de seleção.

\subsection{Processo de Coleta de Dados}

A coleta de dados foi realizada por 3 autores de forma independente (ANC, KSA, SMM), sendo que em caso de discordância, o consenso foi decidido com ajuda de um quarto revisor (DFGO). Para a coleta dos dados, utilizou-se um formulário elaborado no Google Forms. Destaca-se que os dados coletados foram: autores, ano, país, tipo de estudo, critérios diagnósticos, comorbidades, amostra, sexo, idade, histórico médico e valores laboratoriais. Salienta-se que para extrair os dados de proteína C reativa, dímero D e troponina T no estudo de Marfella et al. (2021), foi necessário estimar os valores por meio do software Web Plot Digitizer (versão 4.3) devido os dados estarem disponíveis apenas em gráficos boxplot. Após a coleta dos dados, gerou-se uma planilha no Google Sheets, que possibilitou a tabulação deles no Microsoft Excel. 


\subsection{Avaliação do Risco de Viés}

A avaliação do risco de viés nos estudos incluídos foi realizada com o checklist de avaliação crítica para estudos de coorte do Instituto Joanna Briggs (Aromataris \& Munn, 2020). Este formulário é composto pelas seguintes questões: Q1) Os dois grupos eram semelhantes e recrutados a partir da mesma população? Q2) As exposições foram medidas de forma semelhante para designar as pessoas a grupos expostos e não expostos? Q3) A exposição foi medida de forma válida e confiável? Q4) Foram identificados fatores de confusão? Q5) Foram estabelecidas estratégias para lidar com fatores de confusão? Q6) Os grupos / participantes estavam livres do desfecho no início do estudo (ou no momento da exposição)? Q7) Os resultados foram medidos de forma válida e confiável? Q8) O tempo de follow up foi relatado e suficiente para que os resultados ocorressem? Q9) O follow up foi completo e, em caso negativo, as razões para a perda de acompanhamento foram descritas e exploradas? Q10) Foram utilizadas estratégias para abordar o follow up incompleto? Q11) Foi usada análise estatística apropriada? Vale apontar que a avaliação foi realizada por dois revisores (ANC e SGF), sendo que o consenso foi empreendido por um terceiro revisor (DFGO).

Para classificar o risco geral de viés de cada estudo, utilizamos o percentual de "sim" recebido no checklist. Dessa forma, adotou-se a seguinte classificação: o estudo foi considerado como apresentando alto risco de viés em caso de percentual menor que 50\%; estudo com risco moderado de viés no caso de percentual de 50 a menor que $70 \%$; e baixo risco de viés para percentual igual ou maior que 70\%. É importante destacar, que esses pontos de cortes foram adotados com base em estudos de revisões sistemáticas que também utilizaram checklists do Instituto Joanna Briggs (Polmann et al., 2020; Réus et al., 2021). Ressalta-se que a figura de risco de viés foi gerada com o software R na versão 4.0 .5 (pacote dmetar).

\subsection{Medidas de Efeito e Síntese dos Resultados}

Nesta revisão sistemática identificamos dois desenhos de estudos: dois estudos incluídos iniciaram as investigações a partir de pacientes com COVID-19 e analisaram o desenvolvimento de desfechos nestes pacientes, incluído o desenvolvimento de infarto do miocárdio (Bilaloglu et al., 2020; Yadav et al., 2021); nos outros quatro estudos, os pesquisadores avaliam pacientes com infarto do miocárdio, e em seguida buscam identificar aqueles que apresentaram COVID-19 (Choudry et al., 2020; Güler et al., 2021; Marfella et al., 2021; Popovic et al., 2021).

Considerando então esses dois desenhos de estudos, buscou-se identificar os níveis de dímero D, comparando os seguintes grupos: no primeiro desenho de estudo, comparou-se os níveis de dímero D nos pacientes que desenvolveram infarto do miocárdio com aqueles que não desenvolveram essa doença; no segundo desenho de estudo, comparou-se os níveis de dímero D entre o grupo de pacientes que tiveram COVID-19 e o grupo de pacientes que não apresentaram a infecção.

Vale ressaltar que os níveis de dímero D foram expressos na maioria dos estudos como mediana e intervalo interquartílico (IQR). Por conseguinte, foi necessário estimar a média e desvio-padrão desse marcador cujo método utilizado foi o de Wan et al. (2014). Vale apontar que para a realização das meta-análises os valores de $\mathrm{mg} / \mathrm{L}$ e $\mu \mathrm{g} / \mathrm{L}$ foram convertidos previamente para $\mathrm{ng} / \mathrm{mL}$.

Assim, sintetizou-se os dados por meio de meta-análise para diferença média padronizada entre os grupos, visto que os métodos de quantificação de dímero D podem ter sido diferentes entre os estudos. É importante destacar que ainda se agregou por meta-análises os dados referentes à caracterização sociodemográfica e clínica dos pacientes que tiveram COVID19 e que apresentaram infarto do miocárdio. Para todas as meta-análises, utilizou-se o método do inverso da variância. A heterogeneidade estatística entre os estudos foi avaliada através de $\mathrm{I}^{2}$, teste $\mathrm{Q}$ e pelo valor de $\operatorname{tau}^{2}$ (estimado pelo método de Restricted maximum-likelihood). Ressalta-se que o nível de significância assumido para as análises foi de 5\% (p-valor $<0,05$ ). 
Tabela 1: Estratégias de busca utilizadas nas diferentes bases de dados.

\section{PubMed}

(“COVID-19”[Mesh] OR “COVID-19” OR “COVID 19" OR “COVID-19 Virus Disease" OR “COVID 19 Virus Disease" OR "COVID-19 Virus

Diseases" OR “COVID-19 Virus Infection" OR “COVID 19 Virus Infection” OR “COVID-19 Virus Infections" OR "2019-nCoV Infection" OR "2019 nCoV Infection" OR "2019-nCoV Infections" OR "Coronavirus Disease-19" OR "Coronavirus Disease 19" OR "2019 Novel Coronavirus Disease" OR

"2019 Novel Coronavirus Infection" OR "2019-nCoV Disease" OR "2019 nCoV Disease" OR "2019-nCoV Diseases" OR "COVID19” OR "Coronavirus

Disease 2019" OR "SARS Coronavirus 2 Infection" OR "SARS-CoV-2 Infection" OR "SARS CoV 2 Infection" OR "SARS-CoV-2 Infections" OR

"COVID-19 Pandemic" OR "COVID 19 Pandemic" OR "COVID-19 Pandemics") AND ("fibrin fragment D" [Supplementary Concept] OR "fibrin

fragment D" OR "D-dimer fibrin" OR "D-dimer fragments" OR "fibrin fragment D1 dimer" OR "fibrin fragment DD" OR "D-dimer" OR "fibrin fragment

D-dimer") AND ("Myocardial Infarction"[Mesh] OR "Myocardial Infarction" OR "Myocardial Infarctions" OR "Cardiovascular Stroke" OR

"Cardiovascular Strokes" OR "Myocardial Infarct" OR "Myocardial Infarcts" OR "Heart Attack" OR "Heart Attacks" OR "STEMI" OR "ST-elevation myocardial infarction")

\section{Embase}

\#1('COVID-19' OR ‘COVID 19’ OR ‘COVID-19 Virus Disease' OR ‘COVID 19 Virus Disease' OR ‘COVID-19 Virus Diseases' OR ‘COVID-19 Virus Infection' OR ‘COVID 19 Virus Infection' OR ‘COVID-19 Virus Infections' OR ‘2019-nCoV Infection' OR '2019 nCoV Infection’ OR '2019-nCoV Infections' OR ‘Coronavirus Disease-19' OR ‘Coronavirus Disease 19' OR '2019 Novel Coronavirus Disease' OR '2019 Novel Coronavirus Infection' OR

'2019-nCoV Disease' OR ‘2019 nCoV Disease’ OR ‘2019-nCoV Diseases' OR ‘COVID19' OR ‘Coronavirus Disease 2019' OR 'SARS Coronavirus 2 Infection' OR ‘SARS-CoV-2 Infection' OR ‘SARS CoV 2 Infection' OR 'SARS-CoV-2 Infections' OR 'COVID-19 Pandemic' OR ‘COVID 19

Pandemic' OR 'COVID-19 Pandemics') \#2 ('fibrin fragment D' OR 'D-dimer fibrin' OR 'D-dimer fragments' OR 'fibrin fragment D1 dimer' OR 'fibrin

fragment DD' OR 'D-dimer' OR 'fibrin fragment D-dimer') \#3('Myocardial Infarction' OR 'Myocardial Infarctions' OR 'Cardiovascular Stroke' OR

'Cardiovascular Strokes' OR 'Myocardial Infarct' OR ‘Myocardial Infarcts' OR ‘Heart Attack' OR 'Heart Attacks' OR ‘STEMI' OR 'ST-elevation myocardial infarction') \#4 (\#1 AND \#2 AND \#3)

\section{Web of Science}

TÓPICO: (“COVID-19” OR “COVID 19” OR “COVID-19 Virus Disease” OR “COVID 19 Virus Disease” OR “COVID-19 Virus Diseases” OR

"COVID-19 Virus Infection" OR “COVID 19 Virus Infection” OR “COVID-19 Virus Infections" OR "2019-nCoV Infection” OR "2019 nCoV Infection" OR "2019-nCoV Infections" OR “Coronavirus Disease-19" OR "Coronavirus Disease 19" OR "2019 Novel Coronavirus Disease" OR "2019 Novel

Coronavirus Infection” OR “2019-nCoV Disease” OR “2019 nCoV Disease” OR “2019-nCoV Diseases” OR "COVID19” OR "Coronavirus Disease 2019" OR "SARS Coronavirus 2 Infection" OR "SARS-CoV-2 Infection" OR "SARS CoV 2 Infection" OR "SARS-CoV-2 Infections" OR "COVID-19 Pandemic" OR "COVID 19 Pandemic" OR “COVID-19 Pandemics") AND TÓPICO: ("fibrin fragment D" OR "D-dimer fibrin" OR "D-dimer fragments" OR "fibrin fragment D1 dimer" OR "fibrin fragment DD" OR "D-dimer" OR "fibrin fragment D-dimer") AND TÓPICO: ("Myocardial Infarction" OR

"Myocardial Infarctions" OR "Cardiovascular Stroke" OR "Cardiovascular Strokes" OR "Myocardial Infarct" OR "Myocardial Infarcts" OR "Heart Attack" OR "Heart Attacks" OR "STEMI" OR "ST-elevation myocardial infarction")

\section{Scopus}

TITLE-ABS-KEY ( "COVID-19" OR "COVID 19" OR "COVID-19 Virus Disease" OR "COVID 19 Virus Disease" OR "COVID-19 Virus Diseases" OR

"COVID-19 Virus Infection" OR "COVID 19 Virus Infection" OR "COVID-19 Virus Infections" OR "2019-nCoV Infection" OR "2019 nCoV Infection" OR "2019-nCoV Infections" OR "Coronavirus Disease-19" OR "Coronavirus Disease 19" OR "2019 Novel Coronavirus Disease" OR "2019 Novel

Coronavirus Infection" OR "2019-nCoV Disease" OR "2019 nCoV Disease" OR "2019-nCoV Diseases" OR "COVID19" OR "Coronavirus Disease 2019" OR "SARS Coronavirus 2 Infection" OR "SARS-CoV-2 Infection" OR "SARS CoV 2 Infection" OR "SARS-CoV-2 Infections" OR "COVID-19

Pandemic" OR "COVID 19 Pandemic" OR "COVID-19 Pandemics" ) AND TITLE-ABS-KEY ( "fibrin fragment D" OR "D-dimer fibrin" OR "D-dimer fragments" OR "fibrin fragment D1 dimer" OR "fibrin fragment DD" OR "D-dimer" OR "fibrin fragment D-dimer" ) AND TITLE-ABS-KEY (

"Myocardial Infarction" OR "Myocardial Infarctions" OR "Cardiovascular Stroke" OR "Cardiovascular Strokes" OR "Myocardial Infarct" OR "Myocardial Infarcts" OR "Heart Attack" OR "Heart Attacks" OR "STEMI" OR "ST-elevation myocardial infarction" )

\section{Livivo}

(“COVID-19" OR "COVID 19" OR "COVID-19 Virus Disease" OR "COVID 19 Virus Disease" OR "COVID-19 Virus Diseases" OR "COVID-19 Virus Infection" OR "COVID 19 Virus Infection” OR “COVID-19 Virus Infections" OR “2019-nCoV Infection” OR “2019 nCoV Infection” OR "2019-nCoV Infections" OR "Coronavirus Disease-19" OR "Coronavirus Disease 19" OR "2019 Novel Coronavirus Disease" OR "2019 Novel Coronavirus Infection" OR “2019-nCoV Disease” OR “2019 nCoV Disease” OR “2019-nCoV Diseases” OR “COVID19” OR "Coronavirus Disease 2019” OR "SARS Coronavirus 2 Infection" OR "SARS-CoV-2 Infection" OR "SARS CoV 2 Infection" OR "SARS-CoV-2 Infections" OR "COVID-19 Pandemic" OR "COVID 19 Pandemic" OR "COVID-19 Pandemics") AND ("fibrin fragment D" OR "D-dimer fibrin" OR "D-dimer fragments" OR "fibrin fragment D1 dimer" OR "fibrin fragment DD" OR "D-dimer" OR "fibrin fragment D-dimer") AND ("Myocardial Infarction" OR "Myocardial Infarctions" OR

"Cardiovascular Stroke" OR "Cardiovascular Strokes" OR "Myocardial Infarct" OR "Myocardial Infarcts" OR "Heart Attack" OR "Heart Attacks" OR "STEMI" OR "ST-elevation myocardial infarction")

\section{Google Scholar}

((“COVID”) AND (“fibrin fragment D” OR “D-dimer fibrin” OR “D-dimer fragments” OR “fibrin fragment D1 dimer” OR "fibrin fragment DD” OR “Ddimer" OR "fibrin fragment D-dimer") AND ("Myocardial")) filetype:pdf

Fonte: Autores.

\subsection{Avaliação da Certeza da Evidência}

A avaliação da certeza da evidência foi realizada pelo Grading of Recommendations Assessment, Development, and Evaluation (GRADE) (Guyatt et al., 2011). Os critérios considerados neste sistema de classificação são os seguintes: risco de viés, inconsistência, imprecisão, presença de evidência indireta e o viés de publicação. Destaca-se que nos estudos observacionais a certeza da evidência inicia como sendo baixa podendo diminuir de nível a partir dos critérios supracitados, ou subir de nível nos seguintes casos: efeito de grande magnitude, presença de efeito dose-resposta, ou ainda em casos de efeitos confundidores contrários à medida de efeito. Salienta-se que a avaliação da certeza da evidência foi realizada utilizando software GRADEpro online (McMaster University and Evidence Prime Inc.). 


\section{Resultados}

\subsection{Seleção dos Estudos}

Identificou-se 1021 estudos nas bases de dados (veja Figura 1). Desses, 434 eram duplicados. Por conseguinte, 587 foram avaliados na fase 1 de seleção. Nesta fase, 540 estudos foram excluídos. Por conseguinte, 47 estudos foram analisados na fase 2. Desses, 6 artigos satisfizeram os critérios de elegibilidade. 41 estudos foram excluídos nesta fase pelos seguintes motivos: não apresentavam os dados de dímero D para pacientes com infarto do miocárdio e com COVID-19 (27); relatos de casos (10); não avaliaram infarto do miocárdio (3); e revisão de literatura (1).

Figura 1: Diagrama de fluxo da pesquisa bibliográfica e seleção dos estudos.

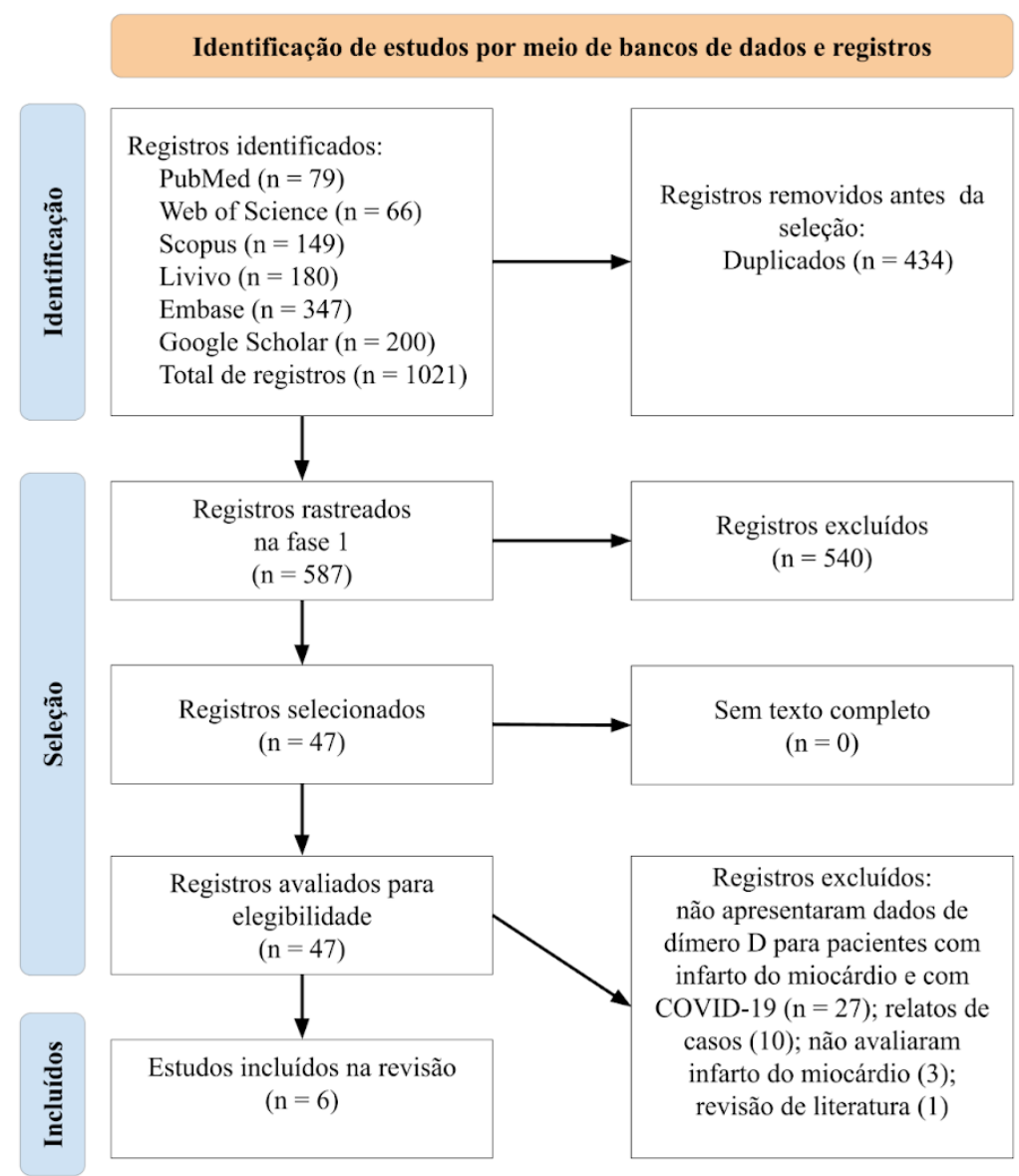

Fonte: Autores.

\subsection{Características dos Estudos}

Nesta revisão sistemática foram incluídos seis estudos, tendo sido os mesmos realizados nos Estados Unidos da América (EUA) (02); Reino Unido (01), Turquia (01), Itália (01), e França (01). Foram publicados entre 2020 e 2021 (veja tabela 2). Todos os estudos eram do tipo coorte, sendo que encontrou-se duas formas de abordagens entre eles: Bilaloglu et al. (2020) e Yadav et al. (2021) iniciaram as investigações a partir de casos de COVID-19 buscando identificar desfechos clínicos como o infarto do miocárdio; Já Choudry et al. (2020), Güler et al. (2021), Marfella et al. (2021) e Popovic et al. (2021) avaliaram se pacientes com infarto do miocárdio haviam ou não apresentado COVID-19.

Na primeira abordagem metodológica, havia um total de 3683 casos de COVID-19, cujo desfecho de infarto do miocárdio ocorreu em 343 pacientes. Vale destacar que desse grupo apenas 3248 pacientes foram incluídos (foram excluídos das análises aqueles pacientes que apresentavam outros desfechos clínicos e aqueles que não apresentavam dados de dímero 
D). Na segunda abordagem metodológica, envolveu-se 500 casos de infarto do miocárdio, sendo que 158 desses pacientes apresentaram exames laboratoriais positivos para COVID-19.

As características sociodemográficas e clínicas dos pacientes que tanto apresentaram COVID-19 e infarto do miocárdio foram agrupadas por meta-análises e podem ser consultadas na Tabela 3. Como podemos perceber, a média de idade desses pacientes foi de 61,09 anos e a maioria dos pacientes era do sexo masculino (68,92\%). Sobre o histórico médico, a maioria apresentava hipertensão arterial sistêmica $(61,47 \%)$, e uma parte considerável dos pacientes apresentava diabetes $(42,01 \%)$ e dislipidemia (37,06\%), sendo que 38,59\% desses pacientes relataram tabagismo. Em relação ao índice de massa corporal (IMC), a média em $\mathrm{kg} / \mathrm{m}^{2}$ foi de 28,11 . E os principais valores laboratoriais disponíveis nos estudos foram: proteína C reativa com média de 50,92 mg/L; 1984,03 ng/mL para dímero D; e 567,42 ng/L para troponina T.

Tabela 2: Resumo das características descritivas dos estudos incluídos.

\begin{tabular}{ccccccc}
\hline Autor & Ano & País & $\begin{array}{c}\text { Tipo de } \\
\text { estudo }\end{array}$ & Amostra & $\begin{array}{c}\text { COVID-19 e } \\
\text { Infarto }\end{array}$ & Valor de dímero D (COVID-19 e Infarto) \\
\hline Bilaloglu et al. & 2020 & EUA & Coorte & 3334 & 298 & Mediana: 546 ng/mL (IQR: 325-1152) \\
Choudry et al. & 2020 & $\begin{array}{c}\text { Reino } \\
\text { Unido }\end{array}$ & Coorte & 115 & 39 & Mediana: $1,86 \mathrm{mg} / \mathrm{L}(\mathrm{IQR}: 0,98-6,6)$ \\
Güler et al. & 2021 & $\begin{array}{c}\text { Turquia } \\
\text { Coorte }\end{array}$ & 126 & 62 & Mediana: $1,20 \mathrm{mg} / \mathrm{L}(\mathrm{IQR}: 1,00-1,70)$ \\
Marfella et al. & 2021 & Itália & Coorte & 176 & 46 & Mediana: $1,782 \mathrm{mg} / \mathrm{L}(\mathrm{IQR}: 1,683-1,995)$ \\
Popovic et al. & 2021 & França & Coorte & 83 & 11 & Média: $3128 \mu \mathrm{Lg} / \mathrm{L}(\mathrm{DP}: 2498)$ \\
Yadav et al. & 2021 & EUA & Coorte & 349 & 45 & Mediana: $2144 \mathrm{ng} / \mathrm{mL}(\mathrm{IQR}: 592-12087)$ \\
\hline
\end{tabular}

DP, desvio padrão; EUA, Estados Unidos da América; IQR, intervalo interquartil. Fonte: Autores.

\subsection{Risco de Viés nos Estudos Incluídos}

Na Figura 2 podemos observar os resultados da avaliação do risco de viés nos estudos incluídos. Em relação ao percentual de "sim" nos itens do checklist, percebe-se que houve uma variação de 66,7 a $100 \%$ entre os estudos. Sobre a avaliação geral do risco de viés, cinco estudos foram classificados como tendo baixo risco de viés e um estudo com risco moderado. Nota-se que os principais problemas apresentados se referem aos relatos sobre o tempo de follow up e à identificação de fatores de confusão. 
Tabela 3: Meta-análises das características sociodemográficas e clínicas dos pacientes que apresentaram COVID-19 e IM.

\begin{tabular}{|c|c|c|c|c|c|}
\hline \multirow{2}{*}{$\begin{array}{c}\text { Característica } \\
\text { (unidade de medida) }\end{array}$} & \multirow[t]{2}{*}{ Estudos* } & \multirow{2}{*}{$\begin{array}{l}\text { Medida de } \\
\text { Efeito }\end{array}$} & \multirow[t]{2}{*}{ IC de $95 \%$} & \multicolumn{2}{|c|}{ Heterogeneidade } \\
\hline & & & & $\mathbf{I}^{2}(\%)$ & $\mathbf{p}$ \\
\hline Idade (anos) & 5 & 61,09 & 57,38 a 64,8 & 86,8 & $<0,0001$ \\
\hline \multicolumn{6}{|l|}{ Sexo } \\
\hline Masculino (\%) & 4 & 68,92 & 57,00 a 80,83 & 72,1 & 0,0131 \\
\hline Feminino (\%) & 4 & 31,08 & 19,17 a 42,99 & 72,1 & 0,0131 \\
\hline \multicolumn{6}{|l|}{ Histórico médico } \\
\hline Diabetes (\%) & 4 & 42,01 & 24,42 a 59,59 & 87,7 & $<0.0001$ \\
\hline HAS $(\%)$ & 5 & 61,47 & 44,70 a 78,23 & 86,3 & $<0.0001$ \\
\hline Dislipidemia (\%) & 4 & 37,06 & 16,48 a 57,65 & 89,1 & $<0.0001$ \\
\hline Tabagismo (\%) & 4 & 38,59 & 13,36 a 63,82 & 95,4 & $<0.0001$ \\
\hline IMC (kg/m²) & 4 & 28,11 & 25,58 a 30,63 & 88,4 & $<0.0001$ \\
\hline \multicolumn{6}{|l|}{ Valores laboratoriais } \\
\hline $\begin{array}{l}\text { Proteína C reativa } \\
(\mathrm{mg} / \mathrm{L})\end{array}$ & 4 & 50,92 & 16,27 a 85,56 & 96,1 & $<0.0001$ \\
\hline Dímero D (ng/mL) & 6 & 1984,03 & 1052,94 a 2915,12 & 99,2 & $<0,0001$ \\
\hline Troponina T (ng/L) & 3 & 567,42 & 151,74 a 2121,93 & 98,2 & $<0.0001$ \\
\hline
\end{tabular}

*Quantidade de estudos incluídos na meta-análise de cada característica. Legenda: IC, intervalo de confiança; IM, infarto do miocárdio; IMC, índice de massa corporal; HAS, hipertensão arterial sistêmica. Fonte: Autores.

Figura 2: Resultados da avaliação do risco de viés nos estudos incluídos.

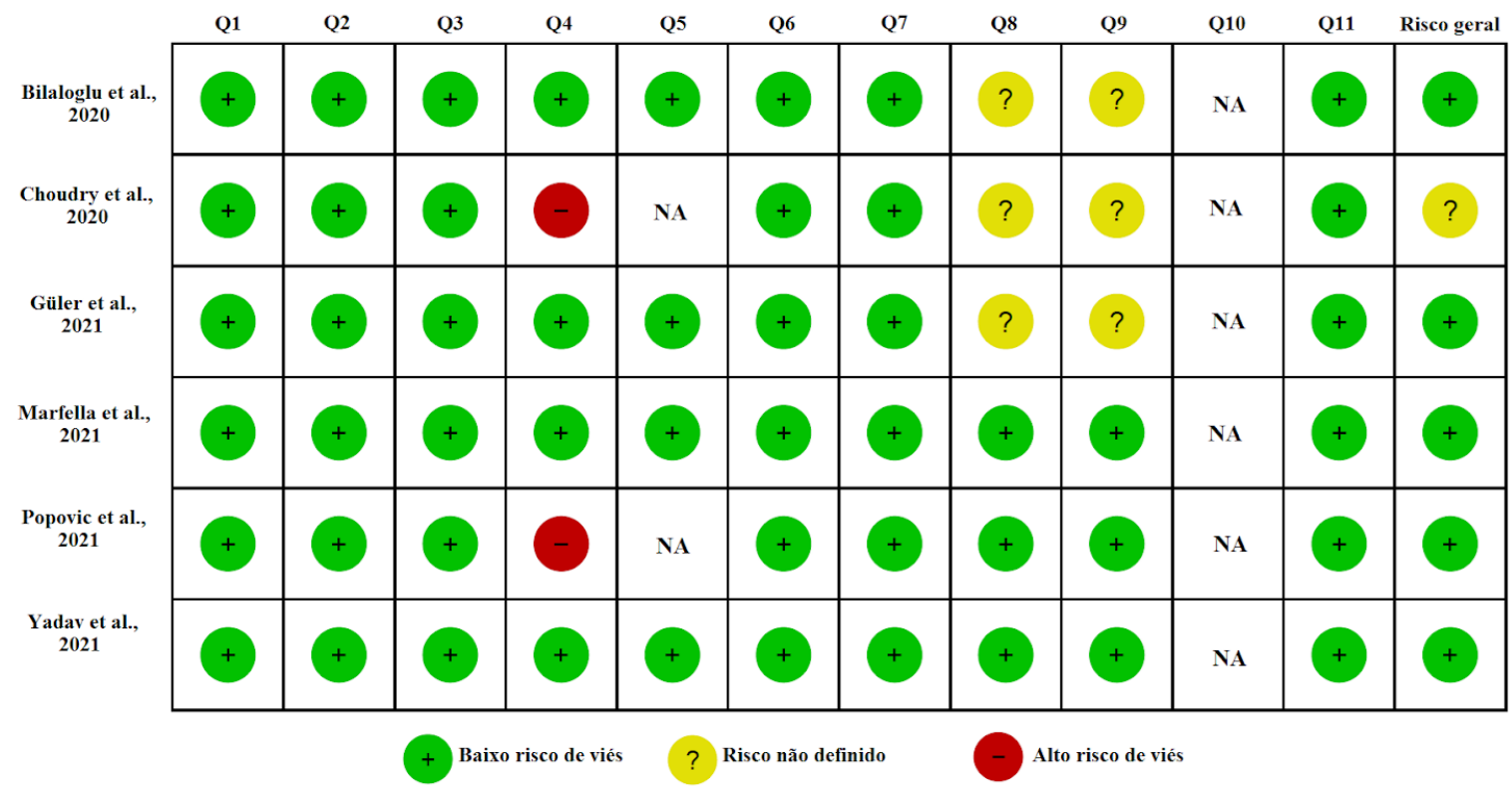

NA, não aplicável. Fonte: Autores.

\subsection{Resultados Individuais dos Estudos}

Em relação a Bilaloglu et al. (2020), pode-se ressaltar que os pesquisadores investigaram 3334 casos de COVID-19, sendo que desses, 298 pacientes apresentaram infarto do miocárdio, 235 casos apresentaram algum outro evento trombótico e 2801 não apresentaram nenhum evento trombótico. Os autores evidenciam no estudo que os níveis de dímero D dentro de 24 horas da admissão foram maiores nos pacientes que desenvolveram infarto [mediana $=546 \mathrm{ng} / \mathrm{mL}$, IQR (325-1152)] do que 
naqueles que não apresentaram nenhum evento trombótico [mediana= $361 \mathrm{ng} / \mathrm{mL}$, IQR (228-622)]. Outro dado importante apresentado no estudo é o fato de valores de dímero D (entre 500 a $1999 \mathrm{ng} / \mathrm{mL}$ no início da admissão ou valores acima) predizer trombose arterial, incluindo nesse grupo dados de infarto do miocárdio [Hazard Ratio (HR): 1,52 (IC95\% de 1,05 a 2,19), $\mathrm{p}=0,03]$.

No estudo de Yadav et al. (2021), que realizaram uma coorte com 349 pacientes com COVID-19, 89 pacientes apresentaram eventos trombóticos, sendo que 45 destes apresentaram infarto do miocárdio. $\mathrm{O}$ estudo evidenciou que os níveis de dímero $\mathrm{D}$ foram maiores nos pacientes que apresentaram tal desfecho em comparação com os pacientes que não apresentaram eventos trombóticos [2144 ng/mL (592-12087) versus 755 (360-1970), p=0,01]. Vale destacar que nesse estudo, os dados de dímero D só estavam disponíveis para 21 pacientes dos 45 que apresentavam infarto do miocárdio, e para 128 pacientes dos 260 que não desenvolveram eventos trombóticos. Nota-se ainda no estudo que valor de dímero D acima de 1000 $\mathrm{ng} / \mathrm{mL}$ foi capaz de predizer eventos trombóticos, incluindo nesses dados os casos de infarto do miocárdio [HR por análise univariada: 3,59 (IC95\% de 1,9 a 7,01), p<0,001; HR por análise multivariada: 2,48 (IC95\% de 1,25 a 5,19), p=0,02].

Acerca da pesquisa realizada por Choudry et al. (2020), podemos afirmar que o grupo investigou 115 casos de infarto do miocárdio com elevação do segmento ST e perceberam que os níveis de dímero D foram maiores nos pacientes que apresentavam COVID-19 do que naqueles que não apresentaram a infecção [mediana (IQR): 1,86 $(0,98-6,6)$ versus $0.52 \mathrm{mg} / \mathrm{L}$ $(0.40-1.0), \mathrm{p}=0.0012]$.

Guler et al. (2021) desenvolveram uma pesquisa observacional com o objetivo de determinar a frequência do fenômeno no-reflow em pacientes com COVID-19 com infarto do miocárdio com elevação de ST (STEMI). Informaram no estudo o resultado da comparação entre os valores médios do dímero D em pacientes que tiveram COVID-19 [mediana (IQR): $1.20 \mathrm{mg} / \mathrm{L}$ (1.00-1.70)] e naqueles que não apresentaram a doença [mediana (IQR): 0,95 (0,80-1,20)], e detectaram diferenças com significância estatística ( $\mathrm{p}<0.001$ ), sendo que os níveis foram maiores no grupo que apresentou COVID-19.

Marfella et al. (2021) investigaram 176 pacientes com STEMI, sendo que 46 desses pacientes apresentaram RT-PCR positivo para SARS-CoV-2. Em relação aos valores de dímero D, os pesquisadores evidenciaram que os níveis desse marcador foram maiores no grupo de pacientes que apresentavam exame de RT-PCR positivo [sem SARS-CoV-2: mediana estimada: 1,00 mg/L (IQR: 0,9-1,1); com SARS-CoV-2: 1,8 (1,7 a 2,0), p<0,01].

E Popovic et al. (2021) investigaram 83 casos de STEMI, nos quais 11 apresentaram COVID-19. Evidenciaram com significância estatística que os valores de dímero D eram maiores nos pacientes que apresentaram a doença viral do que aqueles que não apresentaram a doença [com COVID-19: média (desvio padrão): 3128 g/L (2498); sem COVID-19: 864 (1500), $\mathrm{p}=0,02]$.

\subsection{Síntese dos Resultados}

A figura 3 traz os resultados referente à meta-análise dos níveis de dímero D nos pacientes que tiveram COVID-19 e que desenvolveram ou não infarto do miocárdio. Pode-se notar que a diferença média padronizada entre os pacientes que desenvolveram infarto e aqueles que não tiveram a doença apresentou significância estatística $(p=0,0001)$, sendo que os níveis desse marcador foram maiores nos pacientes que apresentaram infarto do miocárdio. É importante destacar que o agrupamento dos dados não apresentou heterogeneidade com significância estatística.

Outra investigação realizada foi a meta-análise dos níveis de dímero D nos pacientes que tiveram infarto do miocárdio, comparando esses dados entre aqueles que tiveram ou não COVID-19 (veja Figura 4). Observa-se que a diferença média padronizada entre os pacientes com infarto que tiveram COVID-19 e aqueles que não apresentaram tal infecção não evidenciou significância estatística $(\mathrm{p}=0,0512)$. É notável considerar que nesta análise a heterogeneidade estatística entre os estudos foi alta. 
Figura 3: Meta-análise dos níveis de dímero D em pacientes com COVID-19 que desenvolveram ou não IM.

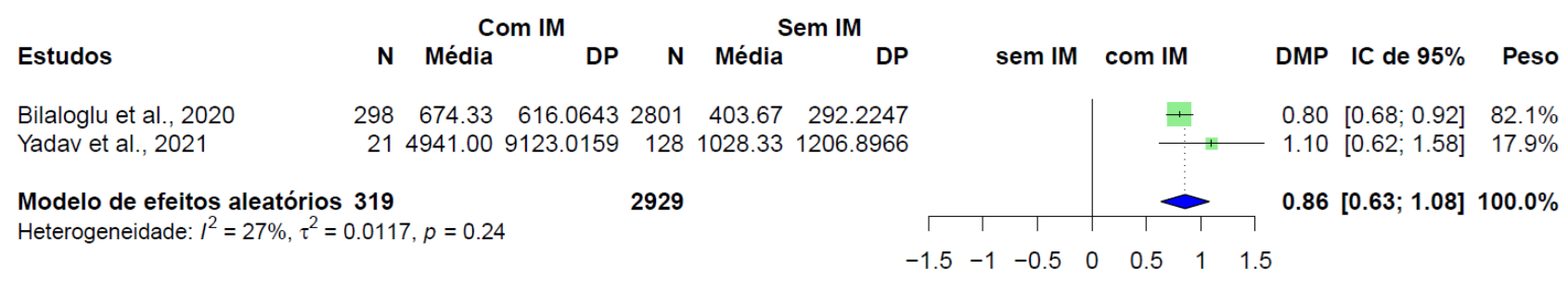

DMP, diferença média padronizada; DP, desvio padrão; IC, intervalo de confiança; IM, infarto do miocárdio; N, tamanho da amostra. Fonte: Autores.

Figura 4: Meta-análise referente aos níveis de dímero D nos pacientes com IM que tiveram ou não COVID-19.

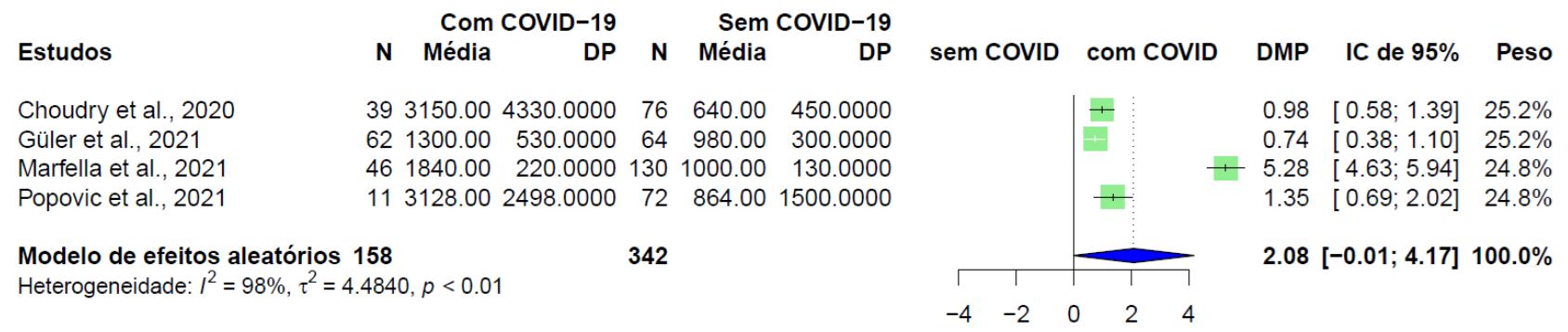

DMP, diferença média padronizada; DP, desvio padrão; IC, intervalo de confiança; IM, infarto do miocárdio; N, tamanho da amostra. Fonte: Autores.

\subsection{Certeza da Evidência}

A tabela 4 traz o resumo dos achados referente a certeza da evidência. Foram avaliados dois desfechos sobre os níveis de dímero $\mathrm{D}$, de acordo com a abordagem metodológica dos estudos e segundo o agrupamento realizado nas meta-análises. Observa-se que a certeza da evidência foi considerada muito baixa para ambos. No caso do desfecho 1, o motivo para tal resultado foi a sugestiva presença de viés de publicação apontado pelo número reduzido de estudos. No caso do desfecho 2 , as causas foram as seguintes: presença de inconsistência apontado pela alta heterogeneidade estatística; presença de evidência indireta, visto que a abordagem metodológica dos estudos incluídos não permitiu responder adequadamente à pergunta da presente revisão; presença de imprecisão evidenciada pelo amplo intervalo de confiança da medida de efeito agrupada na metaanálise; e sugerimos viés de publicação devido o número reduzido de estudos incluídos.

\section{Discussão}

Na presente revisão sistemática, observou-se que os níveis de dímero D foram maiores nos pacientes com COVID-19 que apresentaram infarto do miocárdio do que naqueles que não desenvolveram infarto. Apesar desse dado ter apresentado significância estatística na meta-análise realizada para a primeira abordagem metodológica (figura 3), ele foi classificado como tendo evidência muito baixa, visto que foram incluídos poucos estudos nesta revisão sistemática.

Sobre o dímero D, podemos afirmar que ele é considerado um marcador de coagulação ativada e fibrinólise, sendo que seus níveis refletem a formação e degradação da malha de fibrina ativa (Willeit et al., 2013). Outro ponto importante a destacar é que esse marcador pode estar associado a eventos cardiovasculares subsequentes em pessoas aparentemente saudáveis e em pacientes com angina instável (Choi et al., 2016). 
Tabela 4: Sumário dos achados de acordo com a classificação GRADE

Desfecho 1: níveis de dímero D (entre pacientes com COVID-19 que desenvolveram ou não IM)

\begin{tabular}{|c|c|c|c|c|c|c|}
\hline \multicolumn{7}{|c|}{ Sumário dos resultados: DMP: 0,86 (IC95\% de 0,63 a 1,08), p <0,0001. } \\
\hline $\begin{array}{l}\text { Participantes } \\
\text { (estudos) }\end{array}$ & Risco de viés & INC & $\begin{array}{l}\text { Evidência } \\
\text { indireta }\end{array}$ & Imprecisão & $\begin{array}{c}\text { viés de } \\
\text { publicação }\end{array}$ & $\begin{array}{c}\text { Certeza da } \\
\text { evidência }\end{array}$ \\
\hline $\begin{array}{l}3248 \text { ( } 2 \text { estudos } \\
\text { observacionais) }\end{array}$ & não grave & não grave & não grave & não grave & $\begin{array}{l}\text { altamente } \\
\text { suspeito }^{\mathrm{a}}\end{array}$ & $\begin{array}{l}\text { ÐООО } \\
\text { Muito baixa }\end{array}$ \\
\hline \multicolumn{7}{|c|}{ Desfecho 2: níveis de dímero D (entre pacientes com IM que haviam apresentado ou não COVID-19) } \\
\hline \multicolumn{7}{|c|}{ Sumário dos resultados: DMP: 2,08 (IC95\% de $-0,01$ a 4,17), p=0,0512. } \\
\hline $\begin{array}{l}\text { Participantes } \\
\quad \text { (estudos) }\end{array}$ & Risco de viés & INC & $\begin{array}{l}\text { Evidência } \\
\text { indireta }\end{array}$ & Imprecisão & $\begin{array}{c}\text { viés de } \\
\text { publicação }\end{array}$ & $\begin{array}{c}\text { Certeza da } \\
\text { evidência }\end{array}$ \\
\hline $\begin{array}{c}500 \text { (4 estudos } \\
\text { observacionais) }\end{array}$ & não grave & muito grave ${ }^{b}$ & $\begin{array}{l}\text { muito } \\
\text { grave }^{\mathrm{c}}\end{array}$ & grave $^{\mathrm{d}}$ & $\begin{array}{l}\text { altamente } \\
\text { suspeito }^{\mathrm{a}}\end{array}$ & $\begin{array}{c}\oplus \mathrm{OOO} \\
\text { Muito baixa }\end{array}$ \\
\hline
\end{tabular}

a, número reduzido de estudos. b, alta heterogeneidade estatística; c, a abordagem metodológica não permitiu responder adequadamente à pergunta; d, resultado da meta-análise com intervalo de confiança amplo; DMP, diferença média padronizada; IC, intervalo de confiança; IM, infarto do miocárdio; INC, inconsistência. Fonte: Autores.

No quadro clínico da COVID-19, estudos têm destacado o papel prognóstico deste marcador. Podemos destacar como exemplo, o estudo de Varikasuvu et al. (2021) que realizaram uma revisão sistemática com meta-análises, a qual incluiu 113 estudos. Os autores exploraram o valor prognóstico de dímero D pela análise de estimativas de risco não ajustadas e ajustadas para desfechos associados ao prognóstico de pacientes com COVID-19. Evidenciaram que níveis elevados de dímero D foram capazes de predizer desfechos compostos, a gravidade da doença e a mortalidade desses pacientes.

Shah et al. (2020) também construíram uma revisão sistemática e meta-análise que incluiu 18 estudos, totalizando 3682 pacientes com COVID-19. Evidenciaram que o risco de mortalidade e severidade da doença eram maiores em pacientes com valores de dímero D acima dos valores de referências do que para pacientes com valores normais [risco relativo (RR) de 4,11 para mortalidade (IC95\% de 2,48 a 6,84), p $\leq 0,001$; e RR de 2,04 para severidade da doença (IC95\% de 1,34 a 3,11), $\mathrm{p} \leq 0,001]$.

Outro estudo relevante foi realizado por Gungor et al. (2021). A equipe executou uma meta-análise de 54 estudos, incluindo 11054 pacientes, e observaram que os níveis de dímero D na admissão foram maiores nos pacientes que apresentaram condições clínicas severas, e os níveis também foram maiores naqueles que não sobreviveram à COVID-19.

Sobre a associação do dímero D em pacientes com COVID-19 e infarto do miocárdio, até o presente momento não se identificou outra revisão sistemática. No entanto, muitos estudos têm evidenciado o desenvolvimento de lesões agudas ao miocárdio devido a essa doença viral. É importante destacar que a prevalência dessas lesões é de $20 \%$ (IC95\% de 17 a 23) em pacientes com COVID-19, sendo que os mecanismos envolvidos incluem: hiper-inflamação e tempestade de citocinas; insuficiência respiratória e hipoxemia; regulação negativa da expressão de ACE2 e subsequente vias de sinalização protetora em miócitos cardíacos; hipercoagulabilidade e desenvolvimento de trombose microvascular coronariana; lesão endotelial difusa e "endotelite" no coração; inflamação e/ou estresse causando ruptura da placa coronária ou incompatibilidade ofertademanda levando a isquemia/infarto do miocárdio; e ainda a possibilidade de infiltração direta do miocárdio pelo vírus SARSCoV-2 (Bavishi et al., 2020).

Dentre esses mecanismos, destaca-se a trombose microvascular coronariana. Como o infarto do miocárdio geralmente resulta de trombose oclusiva da placa aterosclerótica, é provável que os níveis circulantes de marcadores que refletem a coagulação ativada e a fibrinólise possam estar associados ao risco de doença cardíaca coronária (Willeit et al., 2013). Dessa forma, Willeit et al. (2013), em uma meta-análise com 18 estudos, evidenciou que existe associação modesta entre dímero D e doença coronariana [RR de 1,23 (IC95\% de 1,16 a 1,32)]. 
Considerando todos esses achados sobre dímero D e infarto do miocárdio, se faz necessário relatar as principais limitações da presente revisão. Uma das limitações foi identificar estudos de coorte que acompanhassem a evolução de pacientes com COVID-19 e investigassem o desfecho infarto do miocárdio. Como podemos perceber nesta revisão, poucos estudos de fato apresentaram essa abordagem metodológica.

Outra limitação importante diz respeito aos dados sobre dímero D. A maioria dos estudos apresentou os dados de dímero-D em forma de mediana e IQR, o que nos levou a estimar a média e desvio padrão. Muitos também não apresentavam dados sobre o fabricante e kit utilizado, e muito menos se os valores de dímero D se encontravam em unidades de dímero D (DDU) ou unidades equivalentes de fibrinogênio (FEU). Outro problema foi a unidade de medida, a qual variou muito entre os estudos. É importante ressaltar que existem mais de 30 ensaios diferentes disponíveis comercialmente para o dímero D e que o antígeno desse marcador não é homogêneo e pode ser detectado de forma diferentes por diferentes ensaios (Favaloro \& Thachil, 2020). Daí a importância de buscar padronizar os métodos de quantificação desse marcador.

Feito essas ressalvas, é importante destacar a importância de analisar esse marcador na prática clínica. Além do dímero D já ser um parâmetro laboratorial bem conhecido e amplamente utilizado para avaliação de eventos trombóticos (Gungor et al., 2021), torna-se importante sua análise nos quadros clínicos de COVID-19, tanto pela capacidade de predizer a progressão da doença, como também pela possibilidade de predizer eventos cardiovasculares, como o infarto do miocárdio. Vale acentuar a necessidade de mais estudos primários que investiguem essa associação, a fim de possibilitar elevação da certeza dessa evidência.

\section{Conclusão}

Nesta revisão sistemática evidenciou-se que existe associação entre os níveis de dímero D em pacientes com COVID19 e infarto do miocárdio. Esse marcador tem se mostrado um bom preditor da progressão da COVID-19, e possivelmente para a ocorrência de infarto do miocárdio. Apesar de significativos, os dados apresentados nesta revisão foram classificados como tendo evidência muito baixa devido o número reduzido de estudos incluídos. Por conseguinte, são necessários mais estudos que busquem investigar o papel do dímero D na ocorrência de infarto do miocárdio em pacientes com COVID-19.

\section{Referências}

Aromataris, E., \& Munn, Z. (Orgs.). (2020). JBI Manual for Evidence Synthesis. JBI. https://doi.org/10.46658/JBIMES-20-01

Bavishi, C., Bonow, R. O., Trivedi, V., Abbott, J. D., Messerli, F. H., \& Bhatt, D. L. (2020). Special Article - Acute myocardial injury in patients hospitalized with COVID-19 infection: A review. Progress in Cardiovascular Diseases, 63(5), 682-689. https://doi.org/10.1016/j.pcad.2020.05.013

Bilaloglu, S., Aphinyanaphongs, Y., Jones, S., Iturrate, E., Hochman, J., \& Berger, J. S. (2020). Thrombosis in Hospitalized Patients With COVID-19 in a New York City Health System. JAMA, 324(8), 799-801. https://doi.org/10.1001/jama.2020.13372

Choi, S., Jang, W. J., Song, Y. B., Lima, J. A. C., Guallar, E., Choe, Y. H., Hwang, J. K., Kim, E. K., Yang, J. H., Hahn, J.-Y., Choi, S.-H., Lee, S.-C., Lee, S. H., \& Gwon, H.-C. (2016). D-Dimer Levels Predict Myocardial Injury in ST-Segment Elevation Myocardial Infarction: A Cardiac Magnetic Resonance Imaging Study. PLOS ONE, 11(8), e0160955. https://doi.org/10.1371/journal.pone.0160955

Choudry, F. A., Hamshere, S. M., Rathod, K. S., Akhtar, M. M., Archbold, R. A., Guttmann, O. P., Woldman, S., Jain, A. K., Knight, C. J., Baumbach, A., Mathur, A., \& Jones, D. A. (2020). High Thrombus Burden in Patients With COVID-19 Presenting With ST-Segment Elevation Myocardial Infarction. Journal of the American College of Cardiology, 76(10), 1168-1176. https://doi.org/10.1016/j.jacc.2020.07.022

Favaloro, E. J., \& Thachil, J. (2020). Reporting of D-dimer data in COVID-19: Some confusion and potential for misinformation. Clinical Chemistry and Laboratory Medicine (CCLM), 58(8), 1191-1199. https://doi.org/10.1515/cclm-2020-0573

Güler, A., Gürbak, İ., Panç, C., Güner, A., \& Ertürk, M. (2021). Frequency and predictors of no-reflow phenomenon in patients with COVID-19 presenting with ST-segment elevation myocardial infarction. Acta Cardiologica, O(0), 1-9. https://doi.org/10.1080/00015385.2021.1931638

Gungor, B., Atici, A., Baycan, O. F., Alici, G., Ozturk, F., Tugrul, S., Asoglu, R., Cevik, E., Sahin, I., \& Barman, H. A. (2021). Elevated D-dimer levels on admission are associated with severity and increased risk of mortality in COVID-19: A systematic review and meta-analysis. The American Journal of Emergency Medicine, 39, 173-179. https://doi.org/10.1016/j.ajem.2020.09.018

Guyatt, G., Oxman, A. D., Ak1, E. A., Kunz, R., Vist, G., Brozek, J., Norris, S., Falck-Ytter, Y., Glasziou, P., \& deBeer, H. (2011). GRADE guidelines: 1. 
Research, Society and Development, v. 11, n. 2, e10211225341, 2022

(CC BY 4.0) | ISSN 2525-3409 | DOI: http://dx.doi.org/10.33448/rsd-v11i2.25341

Introduction-GRADE evidence profiles and summary of findings tables. Journal of Clinical Epidemiology, 64(4), 383-394. https://doi.org/10.1016/j.jclinepi.2010.04.026

Lai, C.-C., Ko, W.-C., Lee, P.-I., Jean, S.-S., \& Hsueh, P.-R. (2020). Extra-respiratory manifestations of COVID-19. International Journal of Antimicrobial Agents, 56(2), 106024. https://doi.org/10.1016/j.ijantimicag.2020.106024

Marfella, R., Paolisso, P., Sardu, C., Palomba, L., D’Onofrio, N., Cesaro, A., Barbieri, M., Rizzo, M. R., Sasso, F. C., Scisciola, L., Turriziani, F., Galdiero, M., Pignataro, D., Minicucci, F., Trotta, M. C., D’Amico, M., Mauro, C., Calabrò, P., Balestrieri, M. L., Paolisso, G. (2021). SARS-COV-2 colonizes coronary thrombus and impairs heart microcirculation bed in asymptomatic SARS-CoV-2 positive subjects with acute myocardial infarction. Critical Care, $25(1), 217$. https://doi.org/10.1186/s13054-021-03643-0

Ouzzani, M., Hammady, H., Fedorowicz, Z., \& Elmagarmid, A. (2016). Rayyan—A web and mobile app for systematic reviews. Systematic Reviews, 5(1). https://doi.org/10.1186/s13643-016-0384-4

Page, M. J., McKenzie, J. E., Bossuyt, P. M., Boutron, I., Hoffmann, T. C., Mulrow, C. D., Shamseer, L., Tetzlaff, J. M., Akl, E. A., Brennan, S. E., Chou, R., Glanville, J., Grimshaw, J. M., Hróbjartsson, A., Lalu, M. M., Li, T., Loder, E. W., Mayo-Wilson, E., McDonald, S., Moher, D. (2021). The PRISMA 2020 statement: An updated guideline for reporting systematic reviews. BMJ, 372, n71. https://doi.org/10.1136/bmj.n71

Polmann, H., Réus, J. C., Maia, I., Dick, B. D., Gozal, D., Flores-Mir, C., Porporatti, A. L., \& De Luca Canto, G. (2020). Association between sleep disordered breathing and symptoms of attention deficits in adults: A systematic review. Sleep Medicine, 73, 223-230. https://doi.org/10.1016/j.sleep.2020.06.031

Popovic, B., Varlot, J., Metzdorf, P. A., Jeulin, H., Goehringer, F., \& Camenzind, E. (2021). Changes in characteristics and management among patients with ST-elevation myocardial infarction due to COVID-19 infection. Catheterization and Cardiovascular Interventions: Official Journal of the Society for Cardiac Angiography \& Interventions, 97(3), E319-E326. https://doi.org/10.1002/ccd.29114

Réus, J. C., Polmann, H., Souza, B. D. M., Flores-Mir, C., Gonçalves, D. A. G., de Queiroz, L. P., Okeson, J., \& De Luca Canto, G. (2021). Association between primary headaches and temporomandibular disorders. The Journal of the American Dental Association, S0002817721004797. https://doi.rgo/10.1016/j.adaj.2021.07.021

Shah, S., Shah, K., Patel, S. B., Patel, F. S., Osman, M., Velagapudi, P., Turagam, M. K., Lakkireddy, D., \& Garg, J. (2020). Elevated d-Dimer Levels Are Associated With Increased Risk of Mortality in Coronavirus Disease 2019: A Systematic Review and Meta-Analysis. Cardiology in Review, 28(6), 295-302. https://doi.org/10.1097/CRD.0000000000000330

Varikasuvu, S. R., Varshney, S., Dutt, N., Munikumar, M., Asfahan, S., Kulkarni, P. P., \& Gupta, P. (2021). D-dimer, disease severity, and deaths (3D-study) in patients with COVID-19: A systematic review and meta-analysis of 100 studies. Scientific Reports, 11(1), 21888. https://doi.org/10.1038/s41598-021$01462-5$

Wan, X., Wang, W., Liu, J., \& Tong, T. (2014). Estimating the sample mean and standard deviation from the sample size, median, range and/or interquartile range. BMC Medical Research Methodology, 14(1), 135. https://doi.org/10.1186/1471-2288-14-135

Willeit, P., Thompson, A., Aspelund, T., Rumley, A., Eiriksdottir, G., Lowe, G., Gudnason, V., \& Di Angelantonio, E. (2013). Hemostatic factors and risk of coronary heart disease in general populations: New prospective study and updated meta-analyses. PloS One, 8(2), e55175. https://doi.org/10.1371/journal.pone.0055175

Yadav, R., Aroshidze, B., Yadav, V., Zahid, U., Jayarangaiah, A., Gandhi, A., \& Gotlieb, V. (2021). Observational Study of Thrombotic Events in a Random Cohort of Hospitalized COVID-19 Patients at a Community-Based Hospital of New York City During the Beginning of the 2020 Pandemic. Cureus, 13(10), e18601. https://doi.org/10.7759/cureus.18601 